\title{
Selenium Modulates the Level of Auxin to Alleviate the Toxicity of Cadmium in Tobacco
}

\author{
Yong Luo ${ }^{1}$, Yuewei Wei ${ }^{1}$, Shuguang Sun ${ }^{2}$, Jian Wang ${ }^{2}$, Weifeng Wang ${ }^{3}$, Dan Han ${ }^{1}$, \\ Huifang Shao ${ }^{1}$, Hongfang Jia ${ }^{1, *}$ and Yunpeng $\mathrm{Fu}^{1, *}$ \\ 1 National Tobacco Cultivation \& Physiology \& Biochemistry Research Centre, College of Tobacco Science, \\ Henan Agricultural University, Zhengzhou 450002, China \\ 2 China Tobacco Hubei Industrial Co., Ltd., Wuhan 430040, China \\ 3 Guangxi Zhuang Autonomous Region Provincial Branch of China National Tobacco Corporation, \\ Nanning 530000, China \\ * $\quad$ Correspondence: jiahongfang@henau.edu.cn (H.J.); yunpengfu@henau.edu.cn (Y.F.); Tel.: +86-371-63555713
}

Received: 23 June 2019; Accepted: 30 July 2019; Published: 1 August 2019

\begin{abstract}
Cadmium (Cd) is an environmental pollutant that potentially threatens human health worldwide. Developing approaches for efficiently treating environmental $\mathrm{Cd}$ is a priority. Selenium (Se) plays important role in the protection of plants against various abiotic stresses, including heavy metals. Previous research has shown that Se can alleviate $\mathrm{Cd}$ toxicity, but the molecular mechanism is still not clear. In this study, we explore the function of auxin and phosphate $(P)$ in tobacco (Nicotiana tabacum), with particular focus on their interaction with Se and Cd. Under Cd stress conditions, low Se $(10 \mu \mathrm{M})$ significantly increased the biomass and antioxidant capacity of tobacco plants and reduced uptake of $\mathrm{Cd}$. We also measured the auxin concentration and expression of auxin-relative genes in tobacco and found that plants treated with low Se $(10 \mu \mathrm{M})$ had higher auxin concentrations at different Cd supply levels $(0 \mu \mathrm{M}, 20 \mu \mathrm{M}, 50 \mu \mathrm{M})$ compared with no Se treatment, probably due to increased expression of auxin synthesis genes and auxin efflux carriers. Overexpression of a high affinity phosphate transporter NtPT2 enhanced the tolerance of tobacco to $\mathrm{Cd}$ stress, possibly by increasing the total $\mathrm{P}$ and Se content and decreasing $\mathrm{Cd}$ accumulation compared to that in the wild type (WT). Our results show that there is an interactive mechanism among P, Se, $\mathrm{Cd}$, and auxin that affects plant growth and may provide a new approach for relieving $\mathrm{Cd}$ toxicity in plants.
\end{abstract}

Keywords: selenium; cadmium stress; auxin; root architecture; phosphate transporter; Nicotiana tabacum

\section{Introduction}

Cadmium (Cd) is a highly toxic heavy metal, which is widely distributed in the environment [1]. In recent years, industrial and agricultural production have discharged $\mathrm{Cd}$ to varying degrees, and it has become one of the most widely distributed agricultural pollutants [2]. Cd is not needed for plant growth and development but is more likely to accumulate in plants than other heavy metals [3]. Evidence suggests that a high concentration of $\mathrm{Cd}$ in soil affects the growth and development of plants through physiological and biochemical processes, including inhibition of plant enzyme and membrane activity [4], decreased cell division [5], reduced growth rate [6], damaged photosynthesis [7], inhibition of stomatal opening [8], and promotion of lipid peroxidation [9]. Cd bioaccumulates and in humans can cause diseases such as osteoporosis, anemia, hypertension, and kidney damage. Soil pollution by $\mathrm{Cd}$ has become a serious threat to the safety of agricultural produce. To address this issue, it is important to develop a comprehensive understanding of the mechanism of Cd uptake in plants. Selenium (Se) is not 
essential for plant nutrition, but it can play a beneficial role in plant health. A suitable dose of Se can enhance antioxidant capacity, delay aging, increase photosynthesis, boost auxin content, and promote plant growth $[10,11]$. By contrast, a high dose of Se can damage plants through reactive oxygen species (ROS) accumulation and inhibit plant growth [12]. Plants are able to utilize soil Se in its inorganic Se (IV) and Se (VI) forms (selenite and selenate, respectively). Studies have shown that plants absorb selenate through sulfate transporters and selenite through phosphate channels [13,14]. A previous study has suggested that Se (IV) uptake is mediated by Pi (inorganic phosphate) transporters [15]. Recent research suggests that auxin is involved in the interaction between Pi and Se in tobacco, which provides convincing evidence for understanding the molecular mechanism of how Se regulates plant growth [16].

Se inhibits Cd uptake, which can relieve the toxic effects of Cd in plants. Cary et al. (1981) first reported that Se fertilizer reduced the absorption of $C d$ in wheat and lettuce [17]. Increasing numbers of studies have shown that there is antagonism between $\mathrm{Cd}$ and Se in plants [18-20]. Cd stress has also been shown to inhibit root growth and leaf photosynthesis in winter wheat, although adding some doses of Se can alleviate the $C d$ toxicity by enhancing root growth [21]. Despite studies investigating the antagonistic relationship between Se and $\mathrm{Cd}$, this relationship-especially the molecular mechanism of Se remission of toxic Cd effects-is still not clear.

Tobacco is an important economic and model crop. In this study, we aimed to clarify the molecular mechanism of Se remission of toxic Cd effects in tobacco. We chose DR5::GUS and NtPT2 overexpressed transgenic tobacco as the test material. We studied the influence of $\mathrm{Cd}$ on growth; the antioxidant system; the auxin distribution; and the $\mathrm{Se}, \mathrm{Cd}$ uptake of tobacco under various $\mathrm{Se}$ and $\mathrm{Cd}$ treatments. We aimed to (1) clarify the function of auxin in tobacco growth, under different doses of Se and Cd; (2) determine the mechanism by which Se enhances tolerance to Cd stress; and (3) reveal the function of phosphate transporter (NtPT2) in the interaction of Se and Cd in plants.

\section{Results}

\subsection{Effects of Se on Tobacco Phenotype and Biomass under Cd Stress}

To determine whether Se affects the growth of tobacco under different $\mathrm{Cd}$ treatments, we checked the characterization of tobacco plants. We found that $\mathrm{Cd}$ treatment had a substantial toxic effect on tobacco seedlings, with greater yellow leaf area and more poorly developed root architecture in Cd-treated seedlings than those in the Cd0 treatment (Figure 1A and Figure S1). Interestingly, we also found that low Se (Se10) could promote the growth of tobacco under Cd stress (Figure 1A); under $\mathrm{Cd} 20$ and Cd50 stress conditions, the biomass of shoots with Se10 treatments increased by $16.3 \%$ and $20.8 \%$, respectively, compared to those with no Se added (Figure 1B), and the biomass of roots with Se10 treatments increased by $24.2 \%$ and $30.2 \%$, respectively (Figure 1C). By contrast, high Se (Se50) levels suppressed tobacco growth under different $\mathrm{Cd}(\mathrm{Cd} 0, \mathrm{Cd} 20$ and $\mathrm{Cd} 50)$ treatments (Figure 1). These results show that Se has a dual effect on Cd stress, and the appropriate concentration of Se can alleviate $\mathrm{Cd}$ toxicity in tobacco. 
A

$\operatorname{Se}(\mathrm{IV})(\mu \mathrm{M})$
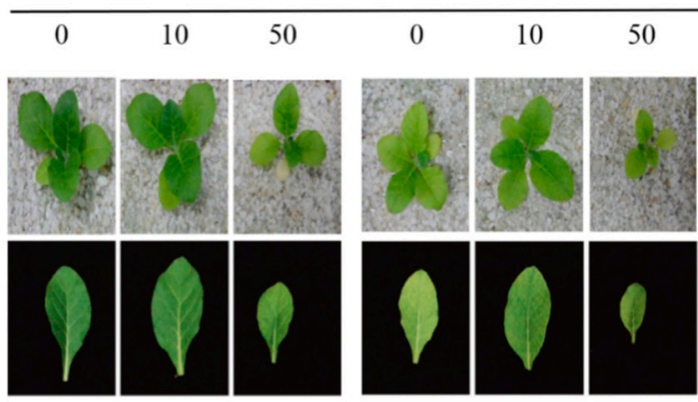

$0 \quad 10 \quad 50$

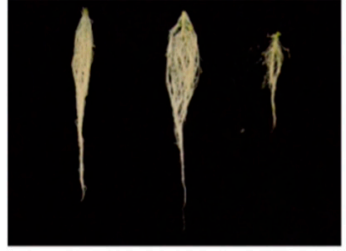

Cd0

B

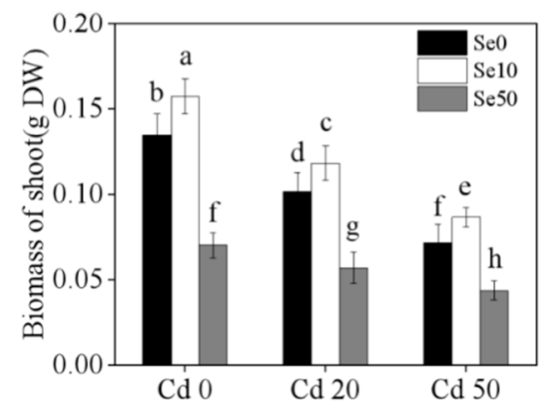

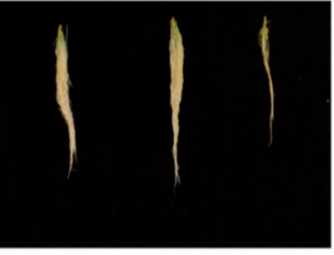

$\mathrm{Cd} 20$
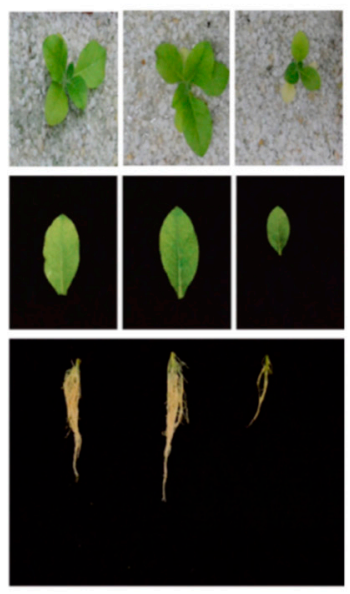

$\mathrm{Cd} 50$

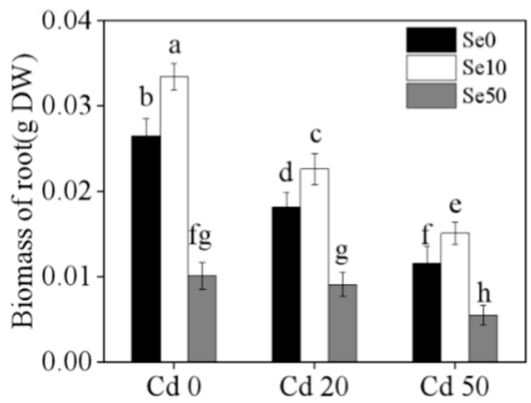

Figure 1. Characterization of tobacco under different Se and Cd concentration supply conditions for 21 days. (A) The phenotype of shoot, third young leaf and root under Se (IV) and Cd (II) treatments in tobacco; (B) The biomass of shoot under different Se (IV) and Cd (II) concentration supply conditions; (C) The biomass of root under different Se (IV) and Cd (II) concentration supply conditions. 14-days-old seedlings (wild-type, Yunyan 87 ) were grown in pots with sand under different Se $(0,10,50 \mu \mathrm{M})$ and Cd $(0,20,50 \mu \mathrm{M})$ concentrations for 21 days. Se0: no Se; Se10: Se, $10 \mu \mathrm{M}$; Se50: Se, $50 \mu \mathrm{M}$; Cd0: no Cd; Cd20: Cd, $20 \mu \mathrm{M}$; Cd50: $50 \mu \mathrm{M}$. Shown are mean \pm standard deviation (SD) from five biological replicates. DW, dry weight. Different letters indicate significant differences $(p<0.05)$.

\subsection{Effects of Se and Cd Interactions on Tobacco Antioxidant Capacity}

Previous studies showed that Se can enhance the enzymatic and non-enzymatic anti-oxidation systems and improve plant resistance to abiotic stresses in plants [4-7]. To determine the function of Se under Cd stress conditions, we measured the antioxidant capacity and chlorophyll content of tobacco. Firstly, we determined the accumulation of $\mathrm{H}_{2} \mathrm{O}_{2}$ by nitroblue tetrazolium (NBT) staining. Low $\mathrm{Se}$ (Se10) obviously reduced the accumulation of $\mathrm{H}_{2} \mathrm{O}_{2}$ (Figure 2A), which was consistent with the results of Malondialdehyde (MDA) content under Cd stress conditions (Figure 2B). We also checked the chlorophyll content, and found that Se had notable effects on chlorophyll content in the leaves (Figure 2C). At no Se (Se0) and high Se (Se50) levels, increased Cd levels significantly reduced tobacco chlorophyll content. Notably, the chlorophyll content of the low Se (Se10) treatment showed a remarkable increase under different $\mathrm{Cd}$ levels, which implies that low Se can promote tobacco growth under $\mathrm{Cd}$ stress by improving the anti-oxidation activity of tobacco plants. 

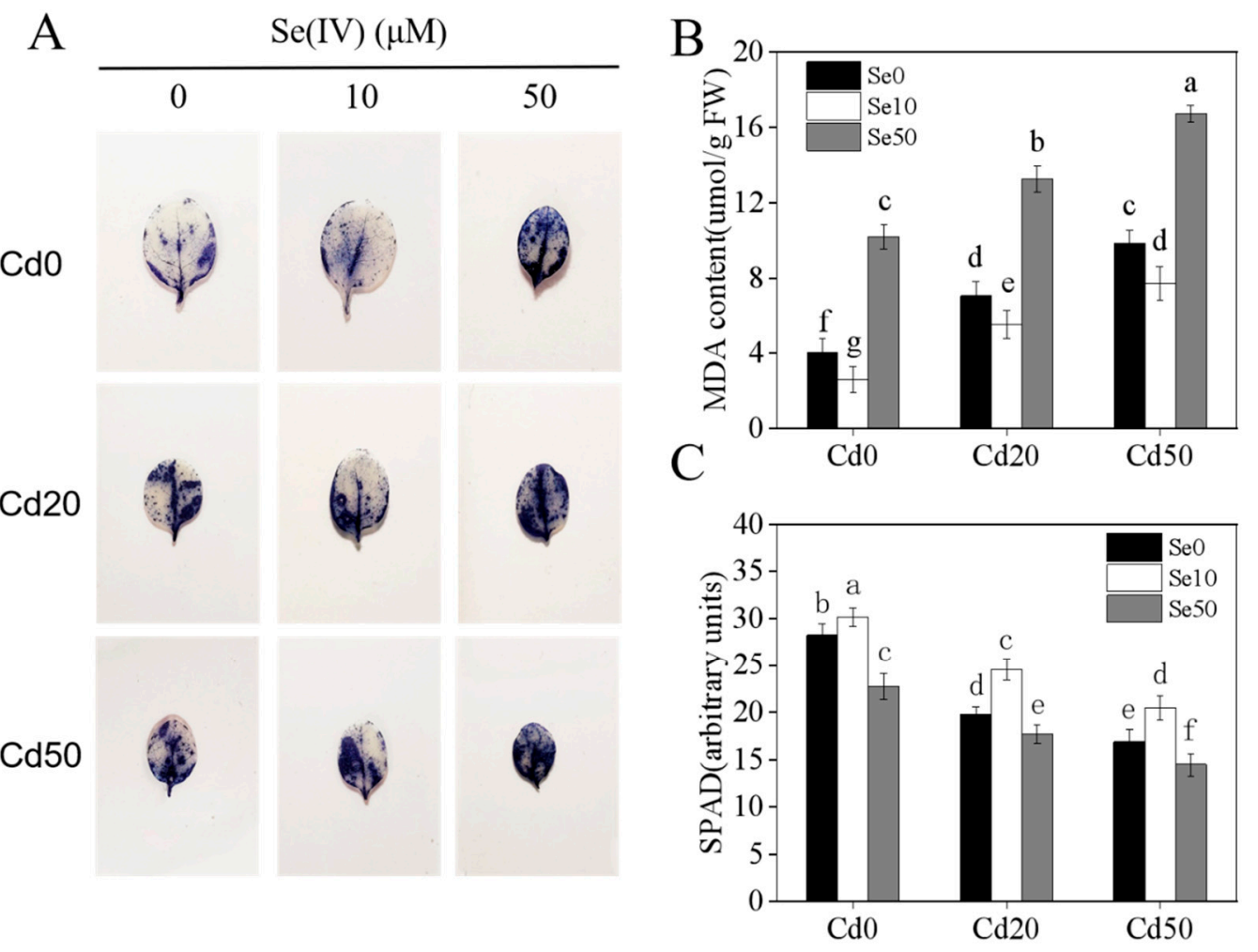

Figure 2. Effects of Se and Cd treatments on antioxidant capacity of tobacco. (A) nitroblue tetrazolium (NBT) staining of tobacco seedlings under different Se (IV) and Cd (II) concentration supply conditions; (B) the content of MDA of tobacco seedlings under different Se (IV) and Cd (II) concentration supply conditions; (C) the SPAD of the third young leaf of tobacco seedlings under different Se (IV) and Cd (II) concentration supply conditions; 14-days-old seedlings (wild-type, Yunyan 87) were grown in pot with sand under different Se $(0,10,50 \mu \mathrm{M})$ and $\mathrm{Cd}(0,20,50 \mu \mathrm{M})$ concentrations for 21 days. Se0: no Se; Se10: Se, $10 \mu \mathrm{M}$; Se50: Se, $50 \mu \mathrm{M}$; Cd0: no Cd; Cd20: Cd, $20 \mu \mathrm{M}$; Cd50: Cd, $50 \mu \mathrm{M}$. Shown are mean \pm SD from five biological replicates. Different letters indicate significant differences $(p<0.05)$.

\subsection{Accumulation of Se and Cd in Tobacco}

To investigate the accumulation of Cd in tobacco under low Se (Se10) and high Se (Se50) conditions, we monitored the Se and Cd content in the roots and shoots of tobacco seedlings (Figure 3). Under high Se and low Se conditions, the Se content of the shoots and roots increased in Cd20 and Cd50 treatments compared with the Cd0 treatment (Figure 3A,B). Under Cd stress (Cd20, Cd50) conditions, the low Se treatment significantly reduced the $\mathrm{Cd}$ content of tobacco shoots and roots, especially under the Cd20 treatment, where the root $\mathrm{Cd}$ content was $37.3 \%$ lower than that observed with the $\mathrm{Se} 0$ treatment (Figure 3C,D). We also found that high Se did not reduce the Cd content of tobacco plants, suggesting that variation in Se content can affect the uptake of $\mathrm{Cd}$ in tobacco. 
A
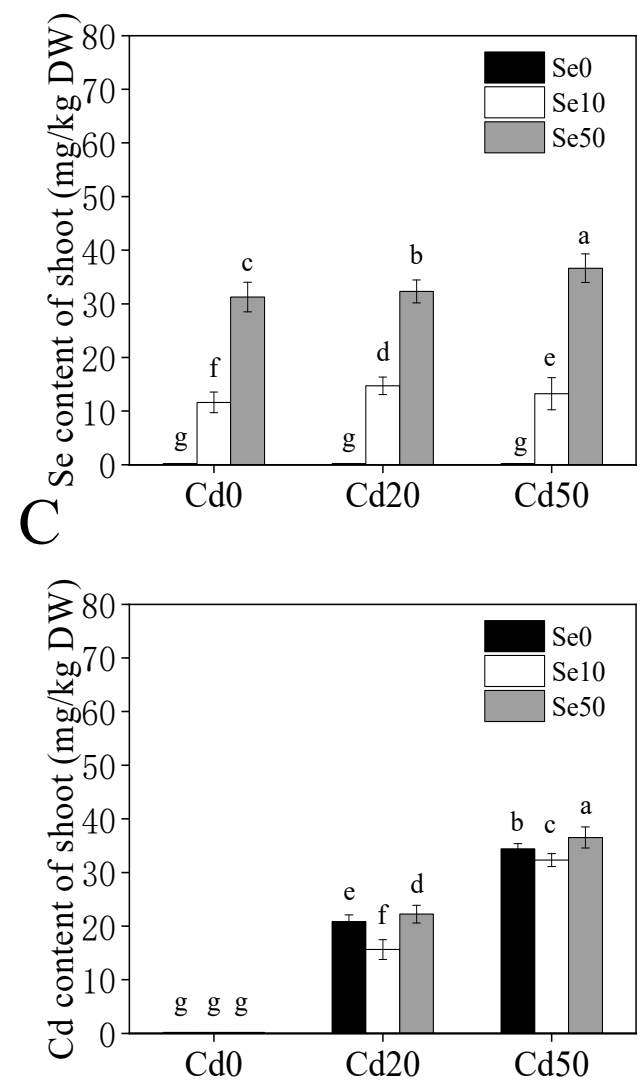

B
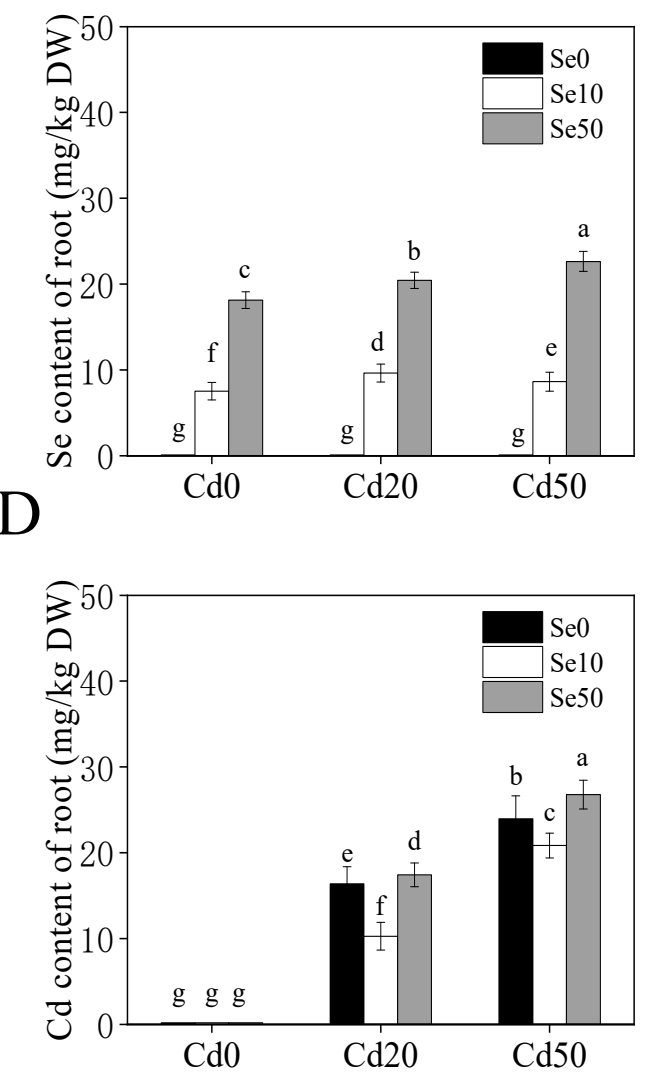

Figure 3. Content of Se and $\mathrm{Cd}$ in tobacco under different Se and $\mathrm{Cd}$ concentration supply conditions for 21 days. (A,B) Se content of the shoots and roots under different Se (IV) and Cd (II) concentration supply conditions; (C,D) Cd content of the shoots and roots under different Se (IV) and Cd (II) concentration supply conditions. 14-days-old seedlings (wild-type, Yunyan 87) were grown in pot with sand under different Se $(0,10,50 \mu \mathrm{M})$ and $\mathrm{Cd}(0,20,50 \mu \mathrm{M})$ concentration for 21 days. Se0: no Se; Se10: Se, $10 \mu \mathrm{M}$; Se50: Se, $50 \mu \mathrm{M}$; Cd0: no Cd; Cd20: Cd, $20 \mu \mathrm{M}$; Cd50: Cd, $50 \mu \mathrm{M}$. Shown are mean \pm SD from five biological replicates. Different letters indicate significant differences $(p<0.05)$.

\subsection{Effects of Se on Auxin and Expression of Auxin-Related Genes in Tobacco under Cd Stress}

To investigate whether auxin is involved in the growth of tobacco roots under Se and Cd treatment, we used DR5::GUS transgenic tobacco, which could reflect the distribution of auxin in the plant [22-24]. We detected GUS expression in the root tip under Se and Cd treatments (Figure 4A). Under Cd stress (Cd20, Cd50) conditions, the GUS expression in the root tip of plants was much lower than observed in the Cd0 treatment. Under low Se (Se10), the expression of GUS in the root tip was much greater than that in Se0 tobacco. Low Se could increase the expression of GUS under Cd stress. We also checked the auxin content of shoots and roots, which was consistent with the GUS expression results (Figure 4B,C). 
A

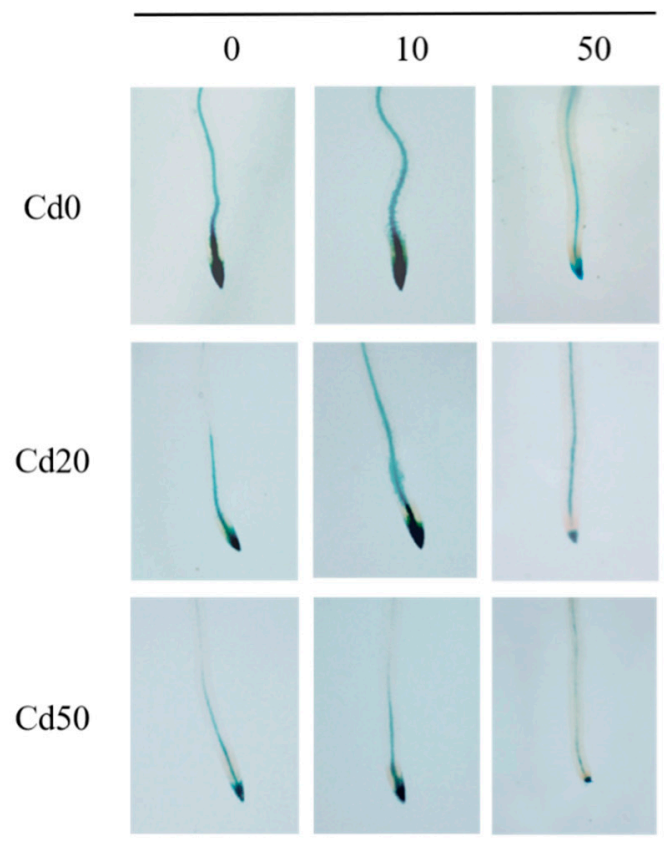

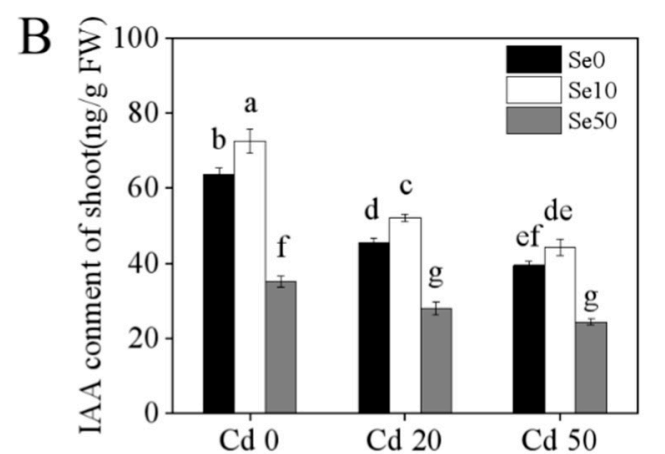

C

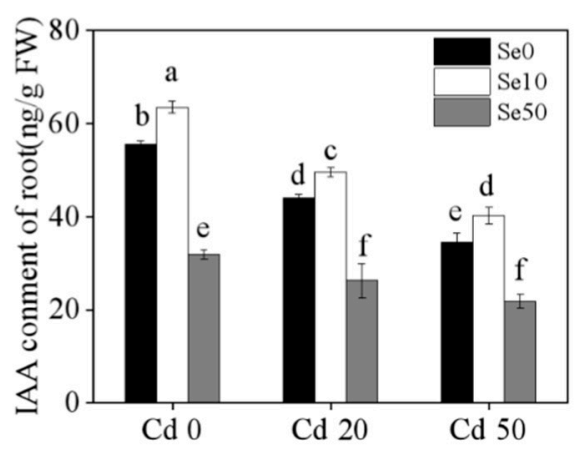

Figure 4. Histochemical localization of DR5::GUS and indole-3-acetic acid (IAA) contents of tobacco under different Se and Cd concentration supply conditions. (A) Histochemical localization of DR5::GUS in root tips of tobacco under different Se (IV) and Cd (II) concentration supply conditions; (B,C) IAA content of the shoots and roots under different Se (IV) and Cd (II) concentration supply conditions. 14-days-old seedlings (DR5::GUS transgenic tobacco) were grown in pot with sand under different Se $(0,10,50 \mu \mathrm{M})$ and $\mathrm{Cd}(0,20,50 \mu \mathrm{M})$ concentrations for 21 days. Se0: no Se; Se10: Se, $10 \mu \mathrm{M}$; Se50: Se, $50 \mu \mathrm{M}$; Cd0: no Cd; Cd20: Cd, $20 \mu \mathrm{M}$; Cd50: Cd, $50 \mu \mathrm{M}$. Shown are mean \pm SD from five biological replicates. Different letters indicate significant differences $(p<0.05)$.

Local auxin levels are determined by biosynthesis and intercellular transport in plant roots [25]. To detect whether Se and Cd treatments affect the auxin-signal pathway, we analyzed the expression of YUCCAs and PINs family genes, which are involved in auxin biosynthesis and transport in tobacco under different Se and Cd treatments. Under Cd stress conditions, the expression of NtYUCCA 6, 8, and 9 , and NtPIN 1a, 1c, and 4 was substantially higher under low Se treatments, which was consistent with the auxin content results (Figure $5 \mathrm{~A}-\mathrm{F}$ ). All these results suggest that auxin may play a key role in growth under Se and Cd treatment conditions. 
A

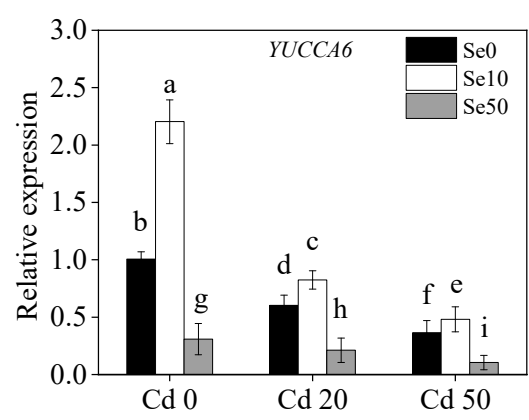

C

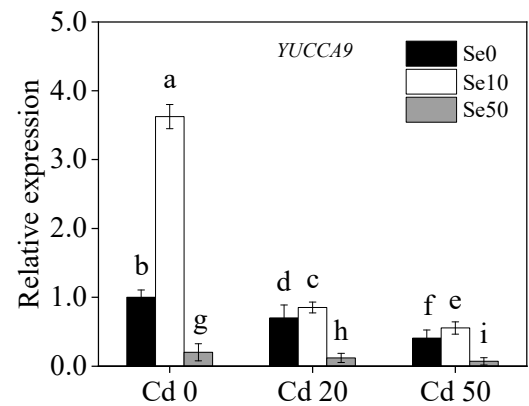

E

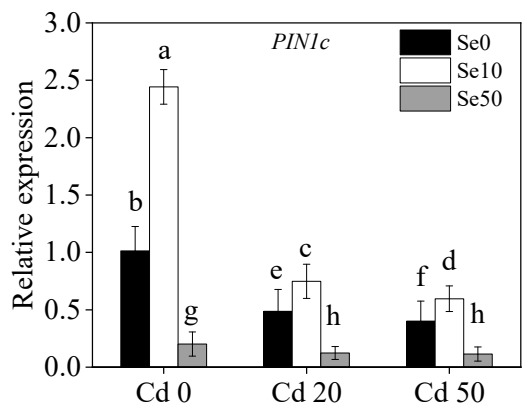

B

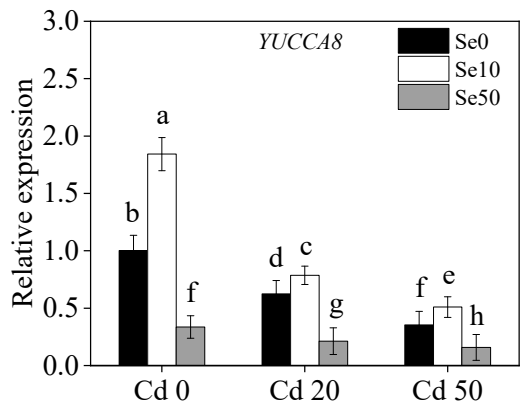

D

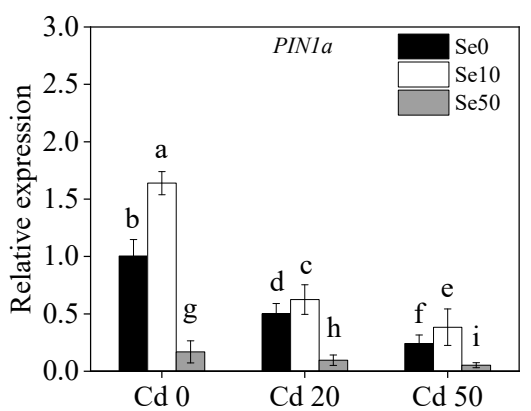

$\mathrm{F}$

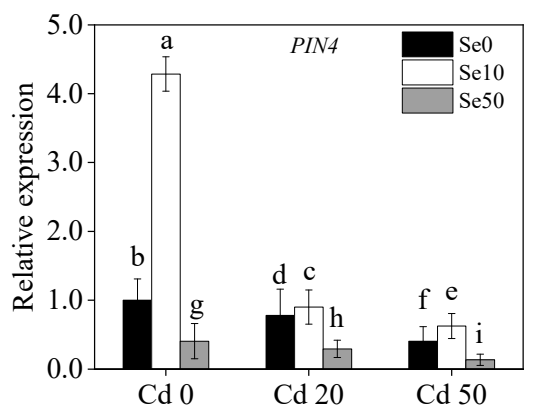

Figure 5. Expression of auxin-relative gene in tobacco under different Se and Cd concentration supply conditions. (A-C) Expression of three members (YUCCA6, 8, 9) of the tobacco YUCCAs family genes in shoots under different $\mathrm{Se}$ and Cd concentration supply conditions.; (D-F) Expression of three members (PIN1a $1 c, 4)$ of the tobacco PINs family genes in roots under different Se and Cd concentration supply conditions. 14-days-old seedlings (DR5::GUS transgenic tobacco) were grown in pot with sand under different $\mathrm{Se}(0,10,50 \mu \mathrm{M})$ and $\mathrm{Cd}(0,20,50 \mu \mathrm{M})$ concentrations for 21 days. The tobacco housekeeping gene $L 25$ was used as an internal control. The relative expression levels are shown compared with the expression under $\mathrm{Cd} 0$ and $\mathrm{Se} 0(\mathrm{Cd} 0+\mathrm{Se} 0)$ conditions as 1 expression. Se0: no Se; Se10: $\mathrm{Se}, 10 \mu \mathrm{M}$; Se50: Se, $50 \mu \mathrm{M}$; Cd0: no Cd; Cd20: Cd, $20 \mu \mathrm{M}$; Cd50: Cd, $50 \mu \mathrm{M}$. Shown are mean \pm SD from five biological replicates. Different letters indicate significant differences $(p<0.05)$.

\subsection{Overexpression of NtPT2 Could Enhance the Tolerance of Cd Stress under Low Se Conditions}

Phosphate transporters are not only involved in Pi uptake, but also in selenite uptake [14,26]. Recently, we reported that the expression of a high-affinity phosphate transporter (NtPT2) involved in Se uptake in tobacco is induced in the roots and shoots under low Pi conditions [16]. In this study, we used NtPT2 overexpression (NtPT2-Oe, two independent transgenic lines: Oe1 and Oe2) in transgenic tobacco to clarify the molecular mechanism by which Se can alleviate Cd toxicity. We found that NtPT2 overexpression in plants enhanced their tolerance to $\mathrm{Cd}$ stress, with plants exhibiting better roots and shoots than the WT under Cd stress conditions. This was consistent with the auxin content differences between the NtPT2-Oe plant and the WT (Figure 6A,B and Figure S2). We also checked the P, Se, and Cd content in both NtPT2-Oe and WT plants; the total P and Se content of NtPT2-Oe plants was higher 
than that of the WT plants under Cd stress conditions (Figure 6C,D), suggesting that NtPT2 is involved in $\mathrm{P}$ and Se uptake in tobacco. We also found that $\mathrm{Cd}$ content was significantly reduced in NtPT2-Oe plants under $\mathrm{Cd}$ stress conditions (Figure 6E), confirming that overexpression of NtPT2 could enhance the tolerance of tobacco plants to Cd stress.
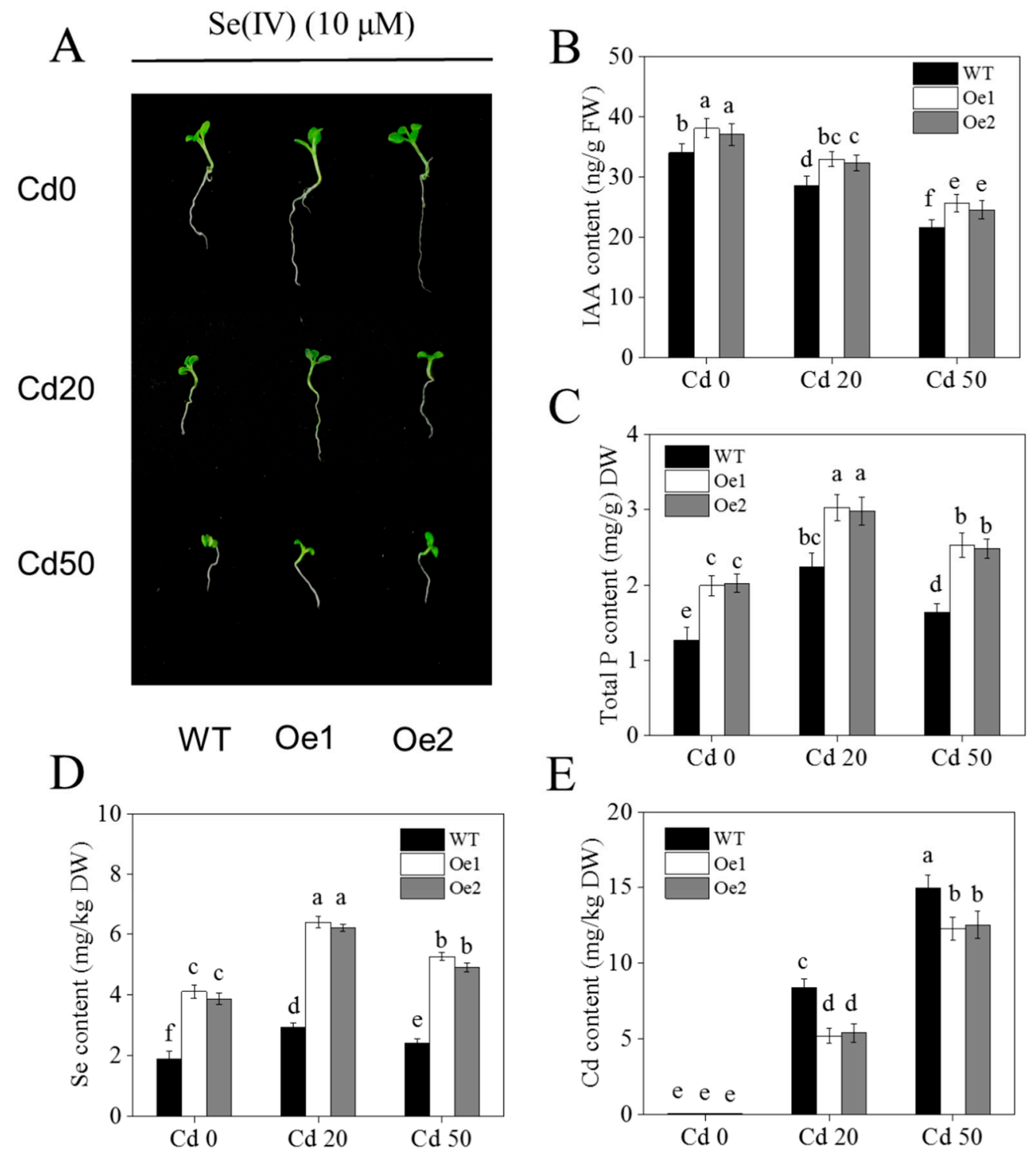

Figure 6. Effects of Cd on NtPT2-Oe transgenic tobacco under different Se and Cd concentration supply conditions. (A,B) The phenotype and IAA content of NtPT2-Oe transgenic tobacco seedlings in Se $(10 \mu \mathrm{M})$ and different $\mathrm{Cd}(0,20,50 \mu \mathrm{M})$ concentrations supply conditions; (C-E) Total P, Se and Cd content of the whole transgenic plant. Tobacco seeds were grown in $1 / 2 \mathrm{MS}$ culture under Se $(10 \mu \mathrm{M})$ and different $C d(0,20,50 \mu \mathrm{M})$ concentrations for 14 days. Se0: no Se; Se10: Se, $10 \mu \mathrm{M}$; Se50: Se, $50 \mu \mathrm{M}$; Cd0: no Cd; Cd20: Cd, $20 \mu \mathrm{M}$; Cd50: Cd, $50 \mu \mathrm{M}$. Shown are mean \pm SD from five biological replicates. Different letters indicate significant differences $(p<0.05)$.

\section{Discussion}

\subsection{Se Affects the Growth of Tobacco Roots by Changing Auxin Concentration under Cd Stress}

$\mathrm{Cd}$ has high levels of biological toxicity and can inhibit the growth and development of plants [27]. Biomass is an important indicator of plant growth and development. In this study, we showed that under $\mathrm{Cd}$ stress conditions, the biomass of tobacco, especially in the roots, was significantly lower than 
plants not exposed to $\mathrm{Cd}$ stresses. This indicates that the roots and shoots were damaged by $\mathrm{Cd}$ stress, which is consistent with the results of Li et al. [28]. We also found that low Se levels stimulated growth in tobacco and could effectively alleviate the toxic effects of Cd stress (Figure 1), which is consistent with the results of previous studies [21,29]. Previous studies have shown that low Se enhances the antioxidation of enzymatic and non-enzymatic systems, changes root growth, and promotes absorption of nutrients [30,31], thus, improving the ability of plants to resist abiotic stresses. Low Se increased anti-oxidation activity (Figure 2), reduced the content of $\mathrm{Cd}$ (Figure 3) and changed root development by increasing auxin concentration in the roots (Figure 4), which may have increased growth. Further analyses on auxin-related genes showed that the expression levels of YUCCAs and NtPINs family genes were markedly higher under low Se conditions (Figure 5), indicating that low Se affects the growth of roots by changing auxin concentration under $\mathrm{Cd}$ stress.

Se often exerts a dual effect on plant growth. High levels of Se cause toxicity in plants, such as accumulation of ROS and inhibition of plant development [12,30,31]. A recent study showed that high Se levels inhibit root growth [16]. In this study, we also found high Se significantly decreased the biomass and auxin content of roots. Notably, under high Se conditions, the Cd treatments significantly decreased the biomass and auxin content in the shoots and roots. To investigate whether auxin is a major regulator of plant growth and development under $\mathrm{Cd}$ stress conditions, we also checked the characterization of root in DR5::GUS transgenic tobacco under different Se and Cd concentration supply conditions by adding IAA $(100 \mathrm{nM})$. The results showed that exogenous IAA could increase the root biomass and the length of primary root, which implies that IAA plays a key roles in regulating the root architecture under $\mathrm{Cd}$ stress conditions (Figure S3).

Altogether, our data suggest that, under Cd stress conditions, Se might affect the growth and development of plants by changing auxin concentration. Not only the auxin pathway, but also other endogenous hormones (e.g., cytokinin, ethylene and gibberellin) may have an effect on plant root development. Recent studies showed that Se increases primary root length through alteration of the auxin and ethylene balance in rice, growth inhibition in Se-treated Arabidopsis is associated with an incomplete mobilization of starch, and high concentrations of selenite-induced enhancement of ethylene biosynthesis may result in plant cell death [32-34]. Therefore, the function of cytokinin, ethylene, gibberellin and other hormones in the development of tobacco roots under the treatment of Se and Cd need further study.

\subsection{NtPT2 Might be a Potential Candidate Gene for Breeding Cd-Tolerant Plants}

A large number of genes encoding Pi transporters have been identified in different plant species. Pi transporters are generally classified into the Pht1, Pht2, Pht3, and PT gene families $[35,36]$. Most of the high-affinity Pi transporter (Pht1) family genes had induced expression under low Pi stress conditions, suggesting that Pht1 plays a crucial role in both Pi uptake and translocation under Pi deficiency $[37,38]$. Recent studies have suggested that Se and Pi share similar uptake mechanisms and that Pi transporters are involved in Se uptake in plants, which might occur via a Se- $\mathrm{H}^{+}$symport process in the plant cell membrane $[14,16,26]$.

Although a large number of Pht1 family genes have been studied in rice and Arabidopsis, the function of key Pi transporters in plants need further research. For instance, recently, two key phosphate transporter genes OsPT2 and OsPT8 were found to play a key role in As and Se uptake and translation in rice, implying that some phosphate transporters are involved in the uptake of heavy metals or trace elements [39]. At present, studies on the Pht1 family gene in tobacco are few. In our previous work, we showed that NtPT2 is the most closely related to OsPT2, and that NtPT2 has similar expression patterns to OsPT2 (low Pi-induced expression in roots) [16]. In this study, we used NtPT2 overexpressing transgenic tobacco to further clarify whether Se could alleviate the toxicity of $\mathrm{Cd}$. Our results showed that when $10 \mu \mathrm{M}$ Se was supplied, the NtPT2 overexpression significantly increased biomass, total P, axuin, and Se content. In contrast, the Cd content in transgenic tobacco obviously reduced under $\mathrm{Cd}$ 
treatments compared with WT (Figure 6), implying that a suitable level of Se modulates the level of auxin, enhancing the tolerance of tobacco to Cd stress.

With the improvement of health awareness, how to reduce the $\mathrm{Cd}$ content in crop production and food chain has become a research hotspot in recent years. It has a potential impact on reducing the accumulation of $\mathrm{Cd}$ in tissues such as liver, kidney, lungs and bones, and avoiding the induction of various diseases such as lung cancer, hypertension and cardiomyopathy $[40,41]$. Our results suggest that NtPT2 might be a potential candidate gene for breeding Cd-tolerant plants, which also need further research.

Based on this study, we propose a possible model for revealing the mechanism controlling the Se, $\mathrm{Cd}$ stress, $\mathrm{P}$, and auxin response in tobacco (Figure 7). Under Cd stress conditions, (1) plant growth is inhibited. (2) Under low Se and Cd stress conditions, the Pi transporter is involved in the uptake of Se (IV) and P in the root, and low Se (accumulation of a small amount Se in plant) changes the auxin-related gene expression, which increases auxin content to promote plant growth. (3) Low Se not only increases the biomass of the root but also enhances the antioxidant capacity. We conclude that low Se alleviates $\mathrm{Cd}$ toxicity in tobacco. However, the function of other hormones in the development of tobacco roots under the treatment of Se and Cd needs further study.

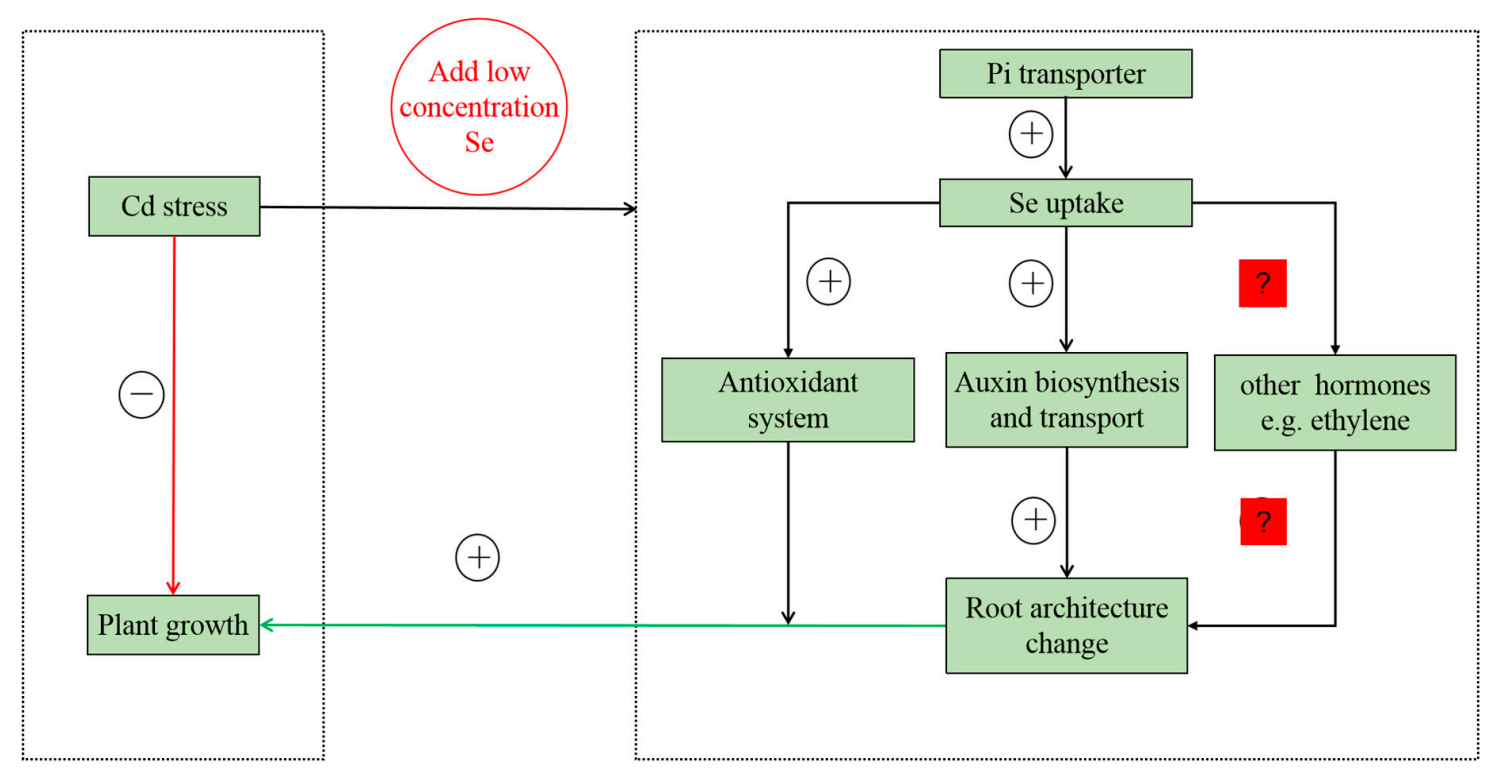

Figure 7. The model for the interaction mechanism between Se, Cd stress, $p$ and auxin response in tobacco. The model is based on the results presented here. + indicates positive regulations and indicates negative regulations. ? indicates that requires further research.

\section{Materials and Methods}

\subsection{Plant Material and Experimental Conditions}

The tested materials were the wild-type (Nicotiana tabacum cv, Yunyan 87), DR5::GUS and NtPT2-Oe transgenic seeds (T2 generation) (Figure $\mathrm{S} 4$ ). The generation of transgenic tobacco material and the construction of $p$ DR5::GUS had been detailed in the previous studies $[16,26,42]$.

Tobacco seeds were sterilized in solution of $75 \%(v / v)$ ethanol for $30 \mathrm{~s}$ and $10 \%(v / v)$ sodium hypochlorite for $7 \mathrm{~min}$, then followed by washing 6 times with sterile distilled water. The seeds were then transferred to seedling tray ( 3 days) kept in the culture room at $28^{\circ} \mathrm{C}$ in dark for proper germination. Germinated seedlings were placed in greenhouse for 10 days. The culture during the first five days used one-fourth-strength Hoagland's nutrient solution, and half-strength nutrient solution was used in the second five days. The tobacco seedlings were transferred in pot with sand and half-strength nutrient solution was used for 4 days in seedling recovering stage. Then they were 
exposed to $\mathrm{Se}\left(\mathrm{Na}_{2} \mathrm{SeO}_{3}\right)$ and $\mathrm{Cd}\left(\mathrm{CdCl}_{2} \cdot 2 \cdot 5 \mathrm{H}_{2} \mathrm{O}\right)$ for 21 days. The experiment of three Se levels, i.e., 0,10 and $50 \mu \mathrm{M}$, and three $\mathrm{Cd}$ levels, i.e., 0,20 and $50 \mu \mathrm{M}$ were designed. There was a total of nine treatments: Cd0+Se0, Cd0+Se10, Cd0+Se50, Cd20+Se0, Cd20+Se10, Cd20+Se50, Cd50+Se0, $\mathrm{Cd} 50+\mathrm{Se} 10$, and $\mathrm{Cd} 0+\mathrm{Se} 50$. Se and $\mathrm{Cd}$ were added into the nutrient solution in the forms of $\mathrm{Na}_{2} \mathrm{SeO}_{3}$ and $\mathrm{CdCl}_{2} \cdot 2 \cdot 5 \mathrm{H}_{2} \mathrm{O}$, respectively.

The tobacco seedings were harvested after 21 days of treatment. Shoots and roots were washed with deionized water for further analysis. (1) We observed and recorded the phenotype of tobacco plants. (2) Some seedings were used to measure the IAA, chlorophyll and MDA content. (3) Some of the leaves and roots were used for NBT and DR5::GUS staining. (4) The other tobacco seedings were oven-dried and used to measure the contents of Se and Cd. (5) Some seedings were used for detecting the gene expression and enzyme activity.

For IAA (Indole-3-acetic acid, dissolved in $1 \mathrm{M} \mathrm{NaOH}$ ) treatments, $100 \mathrm{nM}$ IAA was added to the nutrient solution under different $S e$ and $C d$ treatments, respectively. The nine treatment plants, namely $\mathrm{Cd} 0+\mathrm{Se} 0+\mathrm{IAA}, \mathrm{Cd} 0+\mathrm{Se} 10+\mathrm{IAA}, \mathrm{Cd} 0+\mathrm{Se} 50+\mathrm{IAA}, \mathrm{Cd} 20+\mathrm{Se} 0+\mathrm{IAA}, \mathrm{Cd} 20+\mathrm{Se} 10+\mathrm{IAA}, \mathrm{Cd} 20+\mathrm{Se} 50+\mathrm{IAA}$, $\mathrm{Cd} 50+\mathrm{Se} 0+\mathrm{IAA}, \mathrm{Cd} 50+\mathrm{Se} 10+\mathrm{IAA}$ and $\mathrm{Cd} 50+\mathrm{Se} 50+\mathrm{IAA}$. The tobacco seedings were grown in a growth chamber for 7 days. The plants were then harvested for next stage of analysis. (1) To observe the phenphtype of tobacco plants. (2) To record the histochemical localization of GUS.

\subsection{GUS Staining and Nitroblue Tetrazolium (NBT) Staining of Plant Tissues}

Plants were stained for GUS activity for $24 \mathrm{~h}$ at $37^{\circ} \mathrm{C}$, and then seedlings were immersed in $95 \%$ ethanol to eliminate chlorophyll pigmentation. Plants were stained in NBT solution for $5 \mathrm{~h}$ at $30^{\circ} \mathrm{C}$, and $95 \%$ ethanol was used until decolorization was complete. The stereo microscope (Olympus SZX16, Olympus, Tokyo, Japan) equipped with a colorcharge coupled device (CCD) camera were used to photograph stained plant tissues.

\subsection{Chlorophyll and MDA Measurement}

The relative amount of chlorophyll in plants was determined by a SPAD-502 chlorophyll meter (Konica, Tokyo, Japan) [43]. Five sites of one leaf were measured, and the results were averaged. The content of MDA was determined by the thio-barbituric acid method (TBA) which was calculated by using the difference in absorbance of the extract at $532 \mathrm{~nm}$ and $600 \mathrm{~nm}[44,45]$.

\subsection{IAA Measurement}

The IAA contents of shoots and roots in tobacco seedlings were measured as described by Sun et al. (2014) and Jia et al. (2018) [16,46]. The samples were grinded with appropriate amount of the antioxidant butyleret hydroxytoluen (BHT) and $80 \%$ pre-cooled methanol for $12-16 \mathrm{~h}$. We collected and concentrated the extracted fluid by a rotary evaporator to $10 \mathrm{~mL}$ at $40 \mathrm{~mL}$, and then the fluid was extracted with petroleum ether of the same volume. Under a layer liquid it was adjusted to $\mathrm{pH} 8.5$ and added $0.2 \mathrm{~g}$ polyvinylpyrrolidone (PVP) then vibrated for $30 \mathrm{~min}$, and then filtered through a $0.45 \mu \mathrm{m}$ filter over an OASIS HLB (St. Louis, Mo, USA), and chromatographic conditions were described by: Waters 600-2487; Hibar column RT $250 \times 4.6 \mathrm{~mm}$; Purospher STARRP-18 $(5 \mu \mathrm{m})$; column temperature $45{ }^{\circ} \mathrm{C}$; fluid phase: methanol: $1 \%$ acetic acid $(v / v, 40 / 60)$, isocratic elution; fluid rate: $0.6 \mathrm{~mL} \mathrm{~min}{ }^{-1}$; ultraviolet (UV) detector, $l=269 \mathrm{~nm}$; injection volume $20 \mu \mathrm{L}$. A $0.22 \mu \mathrm{m}$ filter was used for filtration of both the buffer and the samples before high-performance liquid chromatography (HPLC) analysis.

\subsection{Reverse Transcription Polymerase Chain Reaction (qRT-PCR) Analysis}

Trizol reagent was used to prepared Total RNAs from the roots and shoots of tobacco seeding. DNase I-treated total RNAs were used for RT by Superscript II. Triplicate quantitative assays were performed with SYBR Premix Ex Taq ${ }^{\mathrm{TM}}$ II (Perfect Real Time) kit (TaKaRa Biotechnology, Dalian, China) on the Step One Plus RealTime PCR Systems (Applied Biosystems, Bio-Rad, Berkeley, CA USA). The gene-specific primers for YUCCAs and PINs family genes of tobacco were used to perform reverse 
transcription polymerase chain reaction (qRT-PCR) analysis. The primers were shown in Supplemental Table S1. The analysis of relative expression levels used NtL25 (L18908.1) as internal reference gene and presented as $2^{-\Delta \Delta C t}$.

\subsection{Determination of Total P in Plant}

Dry samples of about $0.05 \mathrm{~g}$ were digested with $5 \mathrm{~mL}$ of $98 \% \mathrm{H}_{2} \mathrm{SO}_{4}$ and $3 \mathrm{~mL}$ of $30 \%$ hydrogen peroxide. Then, total $\mathrm{P}$ content was analyzed by the molybdate blue method [47].

\subsection{Measurement of Se and Cd Contents}

The comminuted tobacco samples were digested with concentrated $\mathrm{HNO}_{3}$ and $\mathrm{HClO}_{4}(v / v$, 4:1) [48]. Se and Cd contents were determined by inductively coupled plasma mass spectrometry (ICP-MS 7500A, Agilent, Palo Alto, CA, USA). The accuracy of elemental analysis was verified using standard reference materials from the China Standard Reference Center.

\subsection{Statistical Analysis}

Two-way analysis of variance (ANOVA) and Tukey's multi-comparisons test $(p \leq 0.05)$ were applied to all data. The results were expressed as the means and the corresponding standard errors. All statistical analyses were completed using the Origin2018 (Origin Lab, Northampton, MA, USA) software.

\section{Conclusions}

This study showed that proper Se supply effectively alleviates the toxicity of $\mathrm{Cd}$ in tobacco. Selenium affected the growth of tobacco in the Se-Cd interaction by regulating the expression of the auxin-related genes and enhancing the tolerance to $\mathrm{Cd}$ stress by increasing the content of auxin in tobacco. Overexpression of a high-affinity phosphate transporter NtPT2 increased the content of $\mathrm{P}$ and Se and decreased the accumulation of $\mathrm{Cd}$. This study reveals the interaction mechanism of $\mathrm{P}$ and auxin in plant growth under the action of Se and $\mathrm{Cd}$ and provides new ideas for the safe cultivation of crops in Cd-contaminated soil.

Supplementary Materials: Supplementary materials can be found at http://www.mdpi.com/1422-0067/20/15/ 3772/s1.

Author Contributions: H.J. and Y.F. conceived the research project. Y.L. performed most experiments and wrote the manuscript. Y.W. and W.W. conducted the transgenic tobacco. S.S. and J.W. checked the content of Se and Cd; D.H. and H.S. revised the manuscript. All authors saw and commented on the manuscript.

Funding: This work was supported by the grants from National Natural Science Foundation of China (grant no.31301837) and the Foundation of Henan Educational Committee (grant no.15A210029).

Conflicts of Interest: The authors declare no conflict of interest.

\section{References}

1. Raiesi, F.; Razmkhah, M.; Kiani, S. Salinity stress accelerates the effect of cadmium toxicity on soil N dynamics and cycling: Does joint effect of these stresses matter? Ecotox. Environ. Safe. 2018, 153, 160-167. [CrossRef]

2. Chai, M.W.; Shi, F.C.; Li, R.L.; Liu, L.M.; Liu, Y.; Liu, F.C. Interactive effects of cadmium and carbon nanotubes on the growth and metal accumulation in a halophyte Spartina alterniflora (Poaceae). Plant Growth Regul. 2013, 71, 171-179. [CrossRef]

3. Xue, Y.; Wang, Y.Y.; Yao, Q.H.; Song, K.; Zheng, X.Q.; Yang, J.J. Research progress of plants resistance to heavy metal Cd in soil. Ecol. Environ. Sci. 2014, 23, 528-534.

4. He, X.; Richmond, M.E.; Williams, D.V.; Zheng, W.; Wu, F. Exogenous Glycinebetaine Reduces Cadmium Uptake and Mitigates Cadmium Toxicity in Two Tobacco Genotypes Differing in Cadmium Tolerance. Int. J. Mol. Sci. 2019, 20, 1612. [CrossRef]

5. Zhang, S.S.; Zhang, H.M.; Qin, R.; Jiang, W.S.; Liu, D.H. Cadmium induction of lipid peroxidation and effects on root tip cells and antioxidant enzyme activities inVicia fabaL. Ecotoxicology 2009, 18, 814-823. [CrossRef] 
6. Dias, M.C.; Monteiro, C.; Moutinho-Pereira, J.; Correia, C.; Gonçalves, B.; Conceição, S. Cadmium toxicity affects photosynthesis and plant growth at different levels. Acta Physiol. Plant 2013, 35, 1281-1289. [CrossRef]

7. Parmar, P.; Kumari, N.; Sharma, V. Structural and functional alterations in photosynthetic apparatus of plants under cadmium stress. Bot. Stud. 2013, 54, 45. [CrossRef]

8. Fasahat, P.; Fasahat, P. Advances in Understanding of Cadmium Toxicity and Tolerance in Rice. Emir. J. Food Agr. 2014, 27, 94-105. [CrossRef]

9. Pinto, F.R.; Mourato, M.P.; Sales, J.R.; Moreira, I.N.; Martins, L.L. Oxidative stress response in spinach plants induced by cadmium. J. Plant Nutr. 2017, 40, 268-276. [CrossRef]

10. Kaur, N.; Sharma, S.; Kaur, S.; Nayyar, H. Selenium in agriculture: A nutrient or contaminant for crops? Arch. Agron Soil. Sci. 2014, 60, 1593-1624. [CrossRef]

11. Çakir, Ö.; Turgut-Kara, N.; Ari, Ş. Selenium induced selenocysteine methyltransferase gene expression and antioxidant enzyme activities in Astragalus chrysochlorus. Acta Bot. Croat. 2016, 75, 11-16. [CrossRef]

12. Hartikainen, H.; Xue, T.; Piironen, V. Selenium as an anti-oxidant and pro-oxidant in ryegrass. Plant Soil. 2000, 225, 193-200. [CrossRef]

13. Sors, T.G.; Ellis, D.R.; Salt, D.E. Selenium uptake, translocation, assimilation and metabolic fate in plants. Photosynth. Res. 2005, 86, 373-389. [CrossRef]

14. Zhang, L.H.; Hu, B.; Li, W.; Che, R.H.; Deng, K.; Li, H.; Yu, F.Y.; Ling, H.Q.; Li, Y.J.; Chu, C.C. OsPT2, a phosphate transporter, is involved in the active uptake of selenite in rice. New Phytol. 2014, 201, 1183-1191. [CrossRef]

15. Li, H.F.; Steve, P.M.; Zhao, F.J. Selenium uptake, translocation and speciation in wheat supplied with selenate or selenite. New Phytol. 2008, 178, 92-102. [CrossRef]

16. Jia, H.F.; Song, Z.P.; Wu, F.Y.; Ma, M.; Li, Y.T.; Han, D.; Yang, Y.X.; Zhang, S.T.; Cui, H. Low selenium increases the auxin concentration and enhances tolerance to low phosphorous stress in tobacco. Environ. Exp. Bot. 2018, 153, 127-134. [CrossRef]

17. Cary, E.E. Effect of Selenium and Cadmium Additions to Soil on Their Concentrations in Lettuce and Wheat 1. Agron. J. 1981, 73, 703-706. [CrossRef]

18. He, P.P.; Lv, X.Z.; Wang, G.Y. Effects of Se and Zn Supplementation on the Antagonism against Pb and Cd in Vegetables. Environ. Int. 2004, 30, 167-172. [CrossRef]

19. Saidi, I.; Chtourou, Y.; Djebali, W. Selenium alleviates cadmium toxicity by preventing oxidative stress in sunflower (Helianthus annuus) seedlings. J. Plant Physiol. 2014, 171, 85-91. [CrossRef]

20. Wan, Y.N.; Yu, Y.Y.; Wang, Q.; Qiao, Y.H.; Li, H.F. Cadmium uptake dynamics and translocation in rice seedling: Influence of different forms of selenium. Ecotox. Environ. Safe. 2016, 133, 127-134. [CrossRef]

21. Qin, X.M.; Nie, Z.J.; Liu, H.G.; Zhao, P.; Qin, S.Y.; Shi, Z.W. Influence of selenium on root morphology and photosynthetic characteristics of winter wheat under cadmium stress. Environ. Exp. Bot. 2018, 150, 232-239. [CrossRef]

22. Ulmasov, T.; Murfett, J.; Hagen, G.; Guilfoyle, T.J. Aux/IAA proteins repress expression of reporter genes containing natural and highly active synthetic auxin response elements. Plant Cell. 1997, 9, 1963-1971.

23. Miura, K.; Lee, J.; Gong, Q.Q.; Ma, S.S.; Jin, J.B.; Yoo, C.Y.; Miura, T.; Sato, A.; Bohnert, H.J.; Hasegawa, P.M. SIZ1 Regulation of Phosphate Starvation-Induced Root Architecture Remodeling Involves the Control of Auxin Accumulation. Plant Physiol. 2011, 155, 1000-1012. [CrossRef]

24. Sabatini, S.; Beis, D.; Wolkenfelt, H.; Murfett, J.; Guilfoyle, T.; Malamy, J.; Benfey, P.; Leyser, O.; Bechtold, N.; Weisbeek, P.; et al. An auxin-dependent distal organizer of pattern and polarity in the Arabidopsis root. Cell 1999, 99, 463-472. [CrossRef]

25. Ding, Z.; Jirí, F. Auxin regulates distal stem cell differentiation in Arabidopsis roots. Proc. Natl. Acad. Sci. USA 2010, 107, 12046-12051. [CrossRef]

26. Song, Z.P.; Shao, H.F.; Huang, H.G.; Shen, Y.; Wang, L.Z.; Wu, F.Y.; Han, D.; Song, J.Y.; Jia, H.F. Overexpression of the phosphate transporter gene, OsPT8, improves the Pi and selenium contents in, Nicotiana tabacum. Environ. Exp. Bot. 2017, 137, 158-165. [CrossRef]

27. Nedjimi, B.; Daoud, Y. Cadmium accumulation in Atriplex halimus subsp. schweinfurthii and its influence on growth, proline, root hydraulic conductivity and nutrient uptake. Flora (Jena) 2009, 204, 316-324. [CrossRef]

28. Li, H.; Li, X.; Xiang, L.; Zhao, H.M.; Li, Y.W.; Cai, Q.Y.; Zhu, L.; Mo, C.H.; Wong, M.H. Phytoremediation of soil co-contaminated with Cd and BDE-209 using hyperaccumulator enhanced by AM fungi and surfactant. Sci. Total Environ. 2017, 613-614, 447-455. [CrossRef] 
29. Li, L.; Zhou, W.H.; Dai, H.X.; Cao, F.B.; Zhang, G.P.; Wu, F.B. Selenium reduces cadmium uptake and mitigates cadmium toxicity in rice. J. Hazard. Mater. 2012, 235-236, 343-351.

30. Han, D.; Xiong, S.L.; Tu, S.X.; Liu, J.C.; Chen, C. Interactive effects of selenium and arsenic on growth, antioxidant system, arsenic and selenium species of Nicotiana tabacum L. Environ. Exp. Bot. 2015, 117, 12-19. [CrossRef]

31. Ding, Y.Z.; Feng, R.W.; Wang, R.G.; Guo, J.K.; Zheng, X.Q. A dual effect of Se on Cd toxicity: Evidence from plant growth, root morphology and responses of the antioxidative systems of paddy rice. Plant Soil. 2014, 375, 289-301. [CrossRef]

32. Rafael, S.P.M.; Lucas, C.C.; Rodrigo, T.Á.; Thaline, M.P.; Lubia, S.T.; Fred, A.L.B.; Agustín, Z.; Wagner, L.A.; Ribeiro, D.M. Selenium downregulates auxin and ethylene biosynthesis in rice seedlings to modify primary metabolism and root architecture. Planta 2019, 250, 333-345.

33. Ribeiro, D.M.; Silva, J.; Dalton, D.; Cardoso, F.B.; Martins, A.O.; Silva, W.A.; Nascimento, V.L.; Araújo, W.L. Growth inhibition by selenium is associated with changes in primary metabolism and nutrient levels inArabidopsis thaliana. Plant Cell Environ. 2016, 39, 2235-2246. [CrossRef]

34. Lehotai, N.; Kolbert, Z.; Peto, A.; Feigl, G.; Ordog, A.; Kumar, D.; Tari, I.; Erdei, L. Selenite-induced hormonal and signalling mechanisms during root growth of Arabidopsis thaliana L. J. Exp. Bot. 2012, 63, 5677-5687. [CrossRef]

35. Raghothama, K.G. Phosphate transport and signaling. Curr. Opin. Plant Biol. 2000, 3, 182-187. [CrossRef]

36. Rausch, C.; Bucher, M. Molecular mechanisms of phosphate transport in plants. Planta (Berl.) 2002, 216, 23-37. [CrossRef]

37. Yu, J.; Hu, S.; Wang, J.; Wong, G.K.; Li, S.; Liu, B.; Deng, Y.; Dai, L.; Zhou, Y.; Zhang, X.; et al. A Draft Sequence of the Rice Genome (Oryza sativa L. ssp. japonica). Science 2002, 296, 92-100. [CrossRef]

38. Ai, P.H.; Sun, S.B.; Zhao, J.N.; Fan, X.R.; Xin, W.J.; Guo, Q.; Yu, L.; Shen, Q.R.; Wu, P.; Miller, A.J.; et al. Two rice phosphate transporters, OsPht1;2 and OsPht1;6, have different functions and kinetic properties in uptake and translocation. Plant J. 2009, 57, 798-809. [CrossRef]

39. Wang, P.; Zhang, W.; Mao, C.; Xu, G.; Zhao, F.J. The role of OsPT8 in arsenate uptake and varietal difference in arsenate tolerance in rice. J. Exp. Bot. 2016, 67, 6051-6059. [CrossRef]

40. Xie, L.H.; Xu, Z.R. The toxicity of heavy metal Cadmium to animals and humans. Acta Agric. Zhejianggensis 2003, 6, 52-57. (In Chinese)

41. Prozialeck, W.C.; Edwards, J.R.; Nebert, D.W.; Woods, J.M.; Barchowsky, A.; Atchison, W.D. The Vascular System as a Target of Metal Toxicity. Toxicol. Sci. 2007, 102, 207-218. [CrossRef]

42. Jia, H.F.; Zhang, S.T.; Wang, L.Z.; Yang, Y.X.; Zhang, H.Y.; Cui, H.; Shao, H.F.; Xu, G.H. OsPht1;8, a phosphate transporter, is involved in auxin and phosphate starvation response in rice. J. Exp. Bot. 2017, 68, 5057-5068. [CrossRef]

43. Li, Z.; Tan, X.F.; Lu, K.; Zhang, L.; Long, H.X.; Lv, J.B.; Lin, Q. Influence of drought stress on the growth, leaf gas exchange, and chlorophyll fluorescence in two varieties of tung tree seedlings. Acta Ecol. Sin. 2017, 37, 1515-1524.

44. Feng, R.W.; Wei, C.Y.; Tu, S.X.; Sun, X. Interactive effects of selenium and arsenic on their uptake by Pteris vittata L. under hydroponic conditions. Environ. Exp. Bot. 2009, 65, 363-368. [CrossRef]

45. Han, S.; Fang, L.; Ren, X.J.; Wang, W.L.; Jiang, J. MPK6 controls $\mathrm{H}_{2} \mathrm{O}_{2}$-induced root elongation by mediating $\mathrm{Ca}^{2+}$ influx across the plasma membrane of root cells in Arabidopsis seedlings. New Phytol. 2015, 205, 695-706. [CrossRef]

46. Sun, H.W.; Tao, J.Y.; Liu, S.J.; Huang, S.J.; Chen, S.; Yoneyama, Y.; Zhang, Y.L.; Xu, G.H. Strigolactones are involved in phosphate- and nitrate-deficiency-induced root development and auxin transport in rice. J. Exp. Bot. 2014, 65, 6735-6746. [CrossRef]

47. Chen, A.Q.; Hu, J.; Sun, S.B.; Xu, G.H. Conservation and divergence of both phosphate- and mycorrhiza-regutelad physiological responses and expression patterns of phosphate transporters in solanaceous species. New Phytol. 2007, 173, 817-831. [CrossRef]

48. Wei, C.Y.; Sun, X.; Wang, C.; Wang, W.Y. Factors influencing arsenic accumulation by Pteris vittata: A comparative field study at two sites. Environ. Pollut. 2006, 141, 488-493. [CrossRef]

(C) 2019 by the authors. Licensee MDPI, Basel, Switzerland. This article is an open access article distributed under the terms and conditions of the Creative Commons Attribution (CC BY) license (http://creativecommons.org/licenses/by/4.0/). 